\title{
Healing touch: una opción holística para el cuidado de enfermería
}

\author{
Rosa María Pecina Leyva
}

RESUMEN

La enfermería ha diversificado su campo de acción al incorporar nuevas tendencias de salud, que le permiten estar a la vanguardia y proponer innovadoras actividades que le ayudan al paciente a mejorar su salud; ha desarrollado modelos y paradigmas a partir de diferentes bases conceptuales entre ellas esta implícita la filosofía del holismo, la cual da importancia en comprender al ser humano como una unidad integrando sus componentes: mente, cuerpo y espíritu. Las características de cada paradigma han influido en el desarrollo de la disciplina de enfermería como en las demás disciplinas científicas, que generan un conocimiento autónomo y mantienen continuidad por encima de los cambios en la orientación del conocimiento que la identifican dentro del equipo de salud. Healting touch (HT) tiene la finalidad de lograr la interacción con el paciente para que sea estrecha, comunicando previamente en que consisten sus técnicas y la colaboración que tiene el paciente. Cuando se aplican las técnicas de healing touch seleccionadas para el cuidado del paciente, cada una estan direccionadas por los aspectos éticos. Se dará a conocer la relación que existe entre la enfermería holística, y el healing touch.

Palabras clave: enfermería holística, healing touch, cuidado.

\section{Healing touch: an option for holistic nursing care}

\begin{abstract}
Nursing has diversified its field of action when it incorporated new health trends, which allow it to stay at the vanguard and propose innovative activities that will help patients to improve their health. Nursing has developed models and paradigms from different conceptual bases including implicit philosophy of holism, which gives importance to understanding of human being as a unit, integrating its components: mind, body and spirit. Characteristics of each paradigm have influenced the development of nursing as in other scientific disciplines, generating an independent knowledge and maintaining continuity over changes in knowledge orientation that identified us within a health team. Healing touch (HT) aims to make a closely interaction with patient, previously communicating what Healing Touch techniques are about, and which will be the patient's cooperation. When selected Healing Touch techniques are applied for patient's care, each one is directed by ethical issues. Through this article it will be revealed the relationship between holistic nursing and healing touch.
\end{abstract}

Key words: holistic nursing, healing touch, care.

Correspondencia: Rosa María Pecina Leyva. Universidad Autónoma de San Luis Potosí, S.L.P. México. E-mail: rosypecina@yahoo.com.mx y rosypecina@enfermeria.uaslp.mx

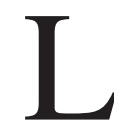

a medicina alternativa es en la actualidad una opción holística para la curación de algunos problemas de salud.

El término medicina alternativa designa de forma amplia métodos y prácticas usados en lugar, o como complemento, de los tratamientos médicos convencionales para curar o paliar enfermedades $^{1-3}$.

La palabra holístico identifica y sugiere terapia integral que genere beneficios tangibles al paciente, implica comprensión y percepción del ser humano como una unidad total formada a partir de la interacción de sus cuerpos: físico, emocional, mental y espiritual $^{4}$.

(HT) incluye un conjunto de técnicas que utilizan el tacto y la compasiva intencionalidad para influir en el sistema de energía de una persona. HT trabaja a través de centros de energía 
como: aura (campo de radiación luminosa alrededor de una persona u objeto como la aureola, en el arte religioso refleja nuestro estado interior), chacras (centros energéticos del cuerpo) que pueden bloquearse y afectar la energía del paciente, los cuales se asocian también con dolor, enfermedad y angustia emocional, revitaliza el sistema energético permitiendo la autosanación en todos los niveles físico, mental, emocional y espiritual $^{5-8}$.

Esta perspectiva del healing touch, da seguimiento terapéutico, ambulatorio u hospitalario, es una intervención para el cuidado de enfermería que favorece el ejercicio independiente con un enfoque holístico.

\section{OBJETIVOS}

Proponer el healing touch, como opción para el cuidado holístico de enfermería ya que permite restaurar la salud a través de armonía y balance del sistema energético del ser humano.

La enfermería holística utiliza modalidades alternativas en la práctica clínica para atender al paciente. La palabra holístico proviene de las raíces griegas olos que significa todo, es la filosofía de la totalidad o integralidad. La introducción de este término en las ciencias de la salud; fué en los años 60's con el auge de la llamada medicina alternativa, practicada en general por personas sin formación médica académica que se autodenominaron médicos holísticos; estos practicantes realizaban técnicas preventivas, diagnósticas y terapéuticas no empleadas por la medicina moderna y propiciaron un enfrentamiento que aún persiste con menor fuerza en la medida que el desarrollo científico comprueba la eficacia de estas técnicas y establece una metodología científica para su aplicación ${ }^{6,7}$. Los límites de la medicina alternativa han cambiado a lo largo del tiempo a medida que algunas técnicas y terapias anteriormente consideradas como alternativas han sido aceptadas por la medicina convencional ${ }^{1}$. Por lo tanto la enfermería modifica su modo de atender las necesidades de nuestra sociedad cambiante.

El método holístico que ahora se describe es healing touch (HT) fue desarrollado por la licenciada en enfermería Janet Mentgen, quien ha estado practicando el manejo de la energía sutil desde 1980 en Denver Colorado. HT se enseña por todo Estados Unidos de Norteamérica, Canadá, Australia, Nueva Zelanda, Inglaterra, India, Alemania, Sudáfrica, e Hispanoamérica $^{3}$.

HT emplea una colección de técnicas energéticas utilizadas en valoración y tratamiento del sistema energético humano, impactando en la salud física, emocional, mental, y espiritual ${ }^{3}$. Para practicar la HT no es necesario ser profesional de la salud pero enfermería ya ha incursionado en esta área y según la
American Nurses Association Holístico Healing Touch apoya, el programa de certificación que se practica en hospitales y clínicas privadas en todo el mundo ${ }^{8}$.

Healing touch complementa otras técnicas de curación, incluyendo la práctica médica convencional en hospitales, clínicas, o técnicas cuerpo-mente orientadas a terapias como masajes, imaginación guiada, musicoterapia, acupresión, la biorretroalimentación, entre otras la psicoterapia HT; no se pretende como una cura, sino como una actividad complementaria 9 .

Healing touch ha documentado beneficios con estudios de investigación relacionados con: neonatos, niños, personas de la tercera edad, con cambios en el sistema inmune, con tratamientos paliativos del dolor, con accidentes vasculares, en pacientes obstétricas, en curación de heridas, entre otros ${ }^{10}$.

Por lo tanto el alcance de la práctica de enfermería holística desde la perspectiva del healing touch, incluye actividades de valoración, diagnóstico, planificación, ejecución de actividades holísticas y evaluación de las respuestas humanas observadas tanto en personas sanas como enfermas, estas respuestas humanas son de naturaleza cambiante dinámica, diversas y exclusivas para cada individuo siendo reflejo de su interacción con el entorno ${ }^{7}$.

\section{CONCLUSIÓN}

La aplicación del healing touch permite proporcionar cuidado holístico de enfermería económico, eficaz y seguro de acuerdo a los problemas identificados en el paciente. HT influye en cambios disfuncionales que impactan a la salud física, emocional, mental, y espiritual favoreciendo el bienestar del individuo al ayudarlo a liberar emociones y sentimientos que le producen inestabilidad emocional. HT ayuda a equilibrar la energía al abarcar la energía biomagnética del individuo, al abordar centros energéticos llamados chakras, lo que favorece la energía positiva en todo el cuerpo del individuo, ayudando a desbloquear todos los canales por donde fluye la energía vital.

La medicina alternativa u holística trata simultáneamente los niveles físico, mental, emocional y espiritual para que el proceso de sanación sea más completo y no sólo se enfoca en la parte afectada, sino en todo su entorno, orientando por último a la persona a que mejore su salud.

En la medida que el healing touch, se aplica como un método terapéutico como el proceso enfermero, se facilitará alcanzar el bienestar holistico, y en esa misma medida se validaran la eficacia de sus postulados. 


\section{REFERENCIAS}

1. es.wikipedia.org/wiki/Medicina_alternativa.

2. www.ombai.com/therapy/summary-of-Holistic...(3)

3. Silva C. Cuaderno de ejercicios de healing touch. Galveston Texas, USA. [EUA]. 2002.

4. www.centroarea.com/joomla/index.php .
5. www.compwellness.net/eGuide/healing.htm

6. bvs.sld.cu/revistas/ems/vol16_4_02/ems07402.htm.(4)

7. bvs.sld.cu/revistas/enf/vol20_2_04/enf07204.htm - 43k -I(5)

8. Hover-Kramer D. El masaje energético curativo. $1^{\mathrm{a}}$. Barcelona: RobinBook. 1999:51-7.

9. www.takingcharge.csh.umn.edu/therapies/healingtouch/what, 9 . www.healingtouchhouston.com

10. www.monografias.com/trabajos $7 /$ meal $/$ meal.shtml 